\title{
The impact of intermittent fasting on body composition and cardiovascular biomarkers: a systematic review and meta-analysis
}

\author{
L. Francis, J. Young and J. Lara \\ Department of Applied Sciences, Faculty of Health and Life Sciences, \\ University of Northumbria at Newcastle, NE1 8ST, UK
}

This abstract was awarded the student prize.

Results of the Health Survey for England 2015 showed that $62.9 \%$ of adults $(67.8 \%$ of men and $58.7 \%$ of women) were overweight or obese $^{(1)}$. Such obesity epidemic requires effective management and prevention strategies to tackle it. Some studies ${ }^{(2)}$ have reported benefits of adopting intermittent fasting, a relatively novel weight loss approach currently attracting media attention. This systematic review and meta-analysis aimed to evaluate the effectiveness of intermittent energy restriction (IER) for weight loss and improvement of blood-borne biomarkers of cardiovascular disease (CVD).

A systematic review of human clinical trials involving IER was undertaken. Web of Science, Medline and Scopus were searched from inception, using specific search terms. A meta-analysis was conducted using RevMan software and statistical heterogeneity was evaluated using the Chi squared and $\mathrm{I}^{2}$ statistic with the $95 \%$ CI for $\mathrm{I}^{2}$ values. The protocol was registered with the International Prospective Register of Systematic Reviews (PROSPERO registration number: CRD42017055434).

The searches yielded 316 publications after removal of duplicates and after screening, 15 papers were included in the qualitative synthesis and nine of these in the meta-analysis. Of the included studies, six originated in North America (USA $n=5$, Canada $\mathrm{n}=1$ ), two in Australia, four in Europe (UK $n=2$, Denmark $n=1$ and Sweden $n=1$ ) and three in Asia (Malaysia $n=3$ ), and involved 746 individuals in total. Meta-analysis showed significant decreases in body fat $(\mathrm{p}=0.02)$ and BMI $(\mathrm{p}=0.05)$, alongside non-significant decreases in body weight $(p=0 \cdot 19)$, waist circumference $(p=0.06)$, total cholesterol $(p=0.40)$, LDL $(p=0 \cdot 27)$, triglycerides $(p=0.08)$, systolic blood pressure $(p=0 \cdot 79)$, diastolic blood pressure $(p=0 \cdot 36)$, serum glucose $(p=0 \cdot 51)$ and serum insulin $(p=0 \cdot 14)$, with non-significant increases in HDL levels $(p=0 \cdot 16)$.

This review identified a small number of trials reporting on IER, highlighting the need for further robust research. Although results are modest, they are in line with other recent reviews and intervention trials showing that IER does have an impact on body composition and blood-borne biomarkers of CVD.

1. PHE. UK and Ireland prevalence and trends (2015) Available from: https://www.noo.org.uk/NOO_about_obesity/adult_obesity/UK_prevalence_ and_trends (Accessed January 2017).

2. Harvie MN, Pegington M, Mattson MP et al. (2011) Int J Obes (Lond). 35, 714-727. 